\title{
Medication overuse headache associated with decreased dopamine transporter availability in the left orbitofrontal cortex: A 11CFT PET/MR study
}

\author{
Huanxian Liu \\ Chinese PLA General Hospital \\ Jiajin Liu \\ Chinese PLA General Hospital
}

\section{Shuping Sun}

Chinese PLA General Hospital

Wei Dai

Chinese PLA General Hospital

\section{Binbin Nie}

Chinese Academy of Agricultural Sciences

\section{Baixuan Xu}

Chinese PLA General Hospital

Zhao Dong ( $\square$ dong_zhaozhao@126.com )

Chinese PLA General Hospital https://orcid.org/0000-0002-4581-2569

\section{Shengyuan Yu}

Chinese PLA General Hospital

\section{Research article}

Keywords: Medication overuse headache, dopamine transporter, orbitofrontal cortex, PET/MR, functional connectivity

Posted Date: October 21st, 2021

DOl: https://doi.org/10.21203/rs.3.rs-968662/v1

License: (1) This work is licensed under a Creative Commons Attribution 4.0 International License. Read Full License 


\section{Abstract}

Backgrounds

The dysfunction of dopamine in the mesocorticolimbic dopamine system in $\mathrm{MOH}$ is unknown. Dopamine transporter (DAT) regulates dopamine clearance and neurotransmission and is sensitive to dopamine levels. A decrease in DAT availability can reflect a decrease in dopamine. To determine DAT availability abnormalities in the mesocorticolimbic dopamine system and explore functional network changes in medication overuse headache $(\mathrm{MOH})$ patients.

Methods

We examined $17 \mathrm{MOH}$ patients and 16 healthy controls (HCs) using integrated positron emission tomography (PET)/magnetic resonance (MR) brain scans with ${ }^{11} \mathrm{CFT}$, a radioligand that binds to DAT. Standardized uptake value ratio (SUVr) images were compared voxelwise between $\mathrm{MOH}$ patients and $\mathrm{HCs}$. Then, the significantly changed cluster ( $p<0.01, \mathrm{GRF}$ correction) with abnormal DAT availability was selected as a specific seed region to further evaluate altered functional connectivity (FC) in $\mathrm{MOH}$. SUVr and mean FC values from significantly changed regions were extracted, and partial correlation analyses with clinical measures were conducted.

Results

$\mathrm{MOH}$ patients had lower SUVr levels in the left rather than right orbitofrontal cortex (OFC) than HCs. There was altered FC between the left OFC and the left superior temporal pole and bilateral calcarine gyri. SUVr levels in the left OFC and the connectivity strength linking the positive brain regions with the left OFC were not correlated with clinical measures in the $\mathrm{MOH}$ patients.

Conclusions

$\mathrm{MOH}$ is characterized by decreased DAT availability in the left OFC, which might reflect compensatory downregulation due to low dopamine signalling within the mesocorticolimbic dopamine system. In addition, the OFC on both sides may have different functions in the pathogenesis of $\mathrm{MOH}$. Altered intrinsic $\mathrm{FC}$ in the left OFC was identified in $\mathrm{MOH}$ patients, which may provide a new perspective to understand the pathogenesis of $\mathrm{MOH}$.

\section{Backgroud}

Medication overuse headache $(\mathrm{MOH})$ is a chronic daily headache caused by frequent ( $\geq 10$ days per month or $\geq 15$ days per month, depending on the medication) and long-term ( $\geq 3$ months) use of medication for acute or symptomatic headache in patients with preexisting primary headache, most commonly migraine [1]. It is recognized as a threat to public health and a disabling condition that causes substantial socioeconomic consequences for society and families [2], ranking 20th in the Global Burden of Disease study in 2015 [3]. 
Although the molecular underpinnings of $\mathrm{MOH}$ remain incompletely understood, various clinical phenomena, such as obsessional drug-taking behaviours, psychological drug dependence or high rates of relapse in $\mathrm{MOH}$ patients [4], have been reported, which may imply a common mechanism shared in $\mathrm{MOH}$ and drug addiction [5]. Moreover, numerous imaging studies with different modalities in the context of drug addiction research have demonstrated structural and functional alterations in the mesocorticolimbic dopamine system [6, 7]. In addition, dopamine is known as the main neurotransmitter involved in the mesocorticolimbic dopamine system [8]. The ability to directly increase dopamine is considered to be a crucial mechanism underlying drug addiction [8]. Therefore, we speculated that dysfunction of dopamine in the mesocorticolimbic dopamine system might play a central role during the development of $\mathrm{MOH}$. However, there are no studies to date exploring dopamine changes in the mesocorticolimbic dopamine system in the context of $\mathrm{MOH}$ research.

The dopamine transporter (DAT) regulates dopamine clearance and neurotransmission and is sensitive to dopamine levels [9]. Clinical and preclinical studies have demonstrated that a decrease in DAT availability was compensatory for the decrease in dopamine signalling $[10,11,12]$ and that dopamine synthesis capacity was positively correlated with DAT availability [13]. Thus, a decrease in DAT availability can reflect a decrease in dopamine.

To test the hypothesis that abnormalities in DAT availability were present in the mesocorticolimbic dopamine system with $\mathrm{MOH}$, we performed combined ${ }^{11} \mathrm{CFT}\left({ }^{11} \mathrm{C}-2 \beta\right.$-carbomethoxy-3 $\beta$-(4-fluorophenyl) tropane, a highly selective radiotracer for DAT) positron emission tomography (PET)/magnetic resonance (MR) imaging in $\mathrm{MOH}$ patients. Then, the cluster with abnormal DAT availability was selected as a specific seed point to further evaluate altered functional connectivity (FC) in $\mathrm{MOH}$.

\section{Methods}

This study was approved by the ethics committee of Chinese PLA General Hospital (S2018-027-02) and registered in the Chinese Clinical Trial Registry (ChicTR1800016773). All subjects provided written informed consent in accordance with the Declaration of Helsinki.

\section{Participants}

Patients who were admitted to the international headache centre in the neurological department of Chinese PLA General Hospital between July 2018 and August 2019 and diagnosed with MOH were potential participants. Patients who agreed to participate in the study were asked to keep a headache diary for at least one month before final enrolment. All enrolled patients were fully evaluated by two headache specialists (Dr. Zhao Dong and Dr. Shengyuan Yu). The inclusion criteria for $\mathrm{MOH}$ were as follows: (1) were 18-65 years of age; (2) had a migraine history before medication overuse; (3) met the $\mathrm{MOH}$ diagnostic criteria based on the International Classification of Headache Disorders-3rd edition (ICHD-3); (4) were without anxiety and depression (i.e., a total score of $>6$ points on the Hamilton Anxiety scale or a total score of $>8$ points on the Hamilton Depression scale); (5) had no sleep disorders (i.e., a total score of $>5$ points on the Pittsburgh Sleep Quality Index); and (6) had not received preventive treatment for $\mathrm{MOH}$ within the 3 months before 
enrolment. The exclusion criteria for the patients with $\mathrm{MOH}$ were as follows: (1) other secondary headaches; (2) comorbid disease requiring long-term use of a pain reliever; and (3) failure to meet the $\mathrm{MOH}$ diagnostic criteria after keeping a headache diary for one month. Healthy controls (HCs) were matched for age and sex. All HCs were recruited by advertisements on WeChat social media and completed a one-month follow-up before final enrolment. The inclusion criteria for HCs were as follows: (1) drug-free and (2) no psychiatric or neurological disorders, including recurrent headache. The exclusion criteria for both groups were pregnancy and contraindications for PET/MR scanning. The study design is outlined in Figure 1.

After PET/MR scans, all MOH patients were prescribed preventive medications, and their intake of acute medication for headache was reduced to $<10$ days/30 days. All enrolled $\mathrm{MOH}$ patients were followed up by telephone or face-to-face consultation. Treatment outcomes were subjectively defined as "satisfactory" when a patient felt $>50 \%$ improved or "poor" when improvement was $<50 \%$ or the patient felt unchanged [14]. Relapse was defined based on the $\mathrm{MOH}$ diagnostic criteria in the ICHD-3.

\section{Image acquisition}

${ }^{11} \mathrm{CFT}$ PET/MR was performed at least 12 hours after the last intake of analgesics. The subjects underwent $\mathrm{PET} / \mathrm{MR}$ after an injection of $3.70 \otimes 5.55 \mathrm{MBq} / \mathrm{kg}{ }^{11} \mathrm{CFT}$. Emission scans were acquired 35 minutes after the injection using an integrated PET/MR scanner (Biograph mMR, Siemens, VB20P). PET images were obtained in list mode for 10 minutes. PET images were reconstructed by a high-definition method with 21 subsets and 3 iterations. Images were postfiltered with a 2-mm Gaussian filter, and the image matrix size was 344. Attenuation correction was performed using UTE $\mu$ map. MR images were acquired on the integrated PET/MR scanner at the same time using a T1-weighted magnetization-prepared rapid acquisition gradient echo (MPRAGE) sequence [repetition time $=1900 \mathrm{~ms}$, echo time $=2.44 \mathrm{~ms}$, flip angle $=9^{\circ}$, field of view $=250 \mathrm{~mm} * 250 \mathrm{~mm}$, matrix $=256 \times 256$, slice thickness $=1.0 \mathrm{~mm}$, and voxel size $=1.0 \times 1.0 \times 1.0 \mathrm{~mm}]$.

Resting-state functional magnetic resonance imaging (fMRI) was obtained using a gradient echo-planar imaging sequence (repetition time $=3000 \mathrm{~ms}$, echo time $=30 \mathrm{~ms}$, flip angle $=90^{\circ}$, slice thickness $=3.0 \mathrm{~mm}$, slice gap $=0$, field of view $=210 \mathrm{~mm} * 210 \mathrm{~mm}$, matrix $=70 \times 70$, and voxel size $=3.0 \times 3.0 \times 3.0 \mathrm{~mm}$ ), and 184 transverse, continuous echo-planar imaging functional volumes were acquired.

\section{Data processing}

Images were analysed using Statistical Parametric Mapping (SPM12 version; Wellcome Department of Cognitive Neurology, Institute of Neurology, London, UK; http//www.fil.ion.ucl.ac.uk/spm) and Data Processing and Analysis for Brain Imaging [15] (DPABI_V6.0_210501) running in MATLAB (version 6.1, Mathworks Inc., Sherborn, MA).

\section{PET image processing}

Images were spatially normalized into Montreal Neurological Institute (MNI) space using 12-parameter affine transformation and subsequent nonlinear registration methods and resliced to $2 \mathrm{~mm}^{3}$. These normalized images were then smoothed using an 8- $\mathrm{mm}^{3}$ full-width at half-maximum (FWHM) Gaussian kernel. The cerebellum was selected as the reference region. DAT availability was measured by the standardized uptake 
value ratio (SUVr). Voxelwise SUVr images were obtained by dividing the SUV value of the cerebellum. Whole-brain voxelwise analysis was conducted between $\mathrm{MOH}$ patients and $\mathrm{HCs}$. The significantly changed regions were generated at the threshold of $P<0.01$ with Gaussian random field (GRF) correction. Furthermore, the mean SUVr of the significantly changed region was calculated.

\section{Resting-state functional connectivity (RSFC)}

The RSFC analysis was performed in DPABI as follows: (1) removal of the first ten volumes; (2) slice timing; (3) spatial normalization; (4) spatial smoothing (FWHM = $8 \mathrm{~mm}$ ) using SPM12; (5) selection of seed regions based on the PET results; and (6) calculation of FC of seed regions using DPABI. The time courses of the seed regions were extracted, and Pearson's correlation coefficients were used to calculate FC between the extracted time course and the averaged time course of the whole brain in a voxelwise manner. (7) The individual r-maps were normalized to Z-maps using Fisher's R-to-Z transformation. (8) Abnormal clusters based on statistical parametric mapping were generated as binary masks, and connectivity strength of the altered brain region was extracted based on Z-maps. Significance was set at $\mathrm{P}<0.05$ with GRF correction. (9) Finally, the mean FC value in each significantly changed region was extracted.

\section{Statistical analysis}

Numerical results are presented as the means $( \pm S D)$ or medians with interquartile ranges. Age was compared with a $t$ test, and sex ratio was compared with Fisher's exact test. The SUVr and mean FC values of the cluster that showed a significant group difference were further extracted to perform partial correlation analysis with clinical measures (headache duration, medication overuse duration, pain intensity, headache frequency, and treatment outcomes). For the clinical measures, partial correlations were run in the $\mathrm{MOH}$ group only. Age and sex were used as covariates in all correlation analyses. These statistics were processed using IBM SPSS 26, and statistical significance was set at $P<0.05$.

\section{Results}

A total of 21 eligible $\mathrm{MOH}$ patients and $17 \mathrm{HCs}$ were enrolled. One patient failed to meet the MOH diagnostic criteria after review of a headache diary that was kept for one month. Three patients failed to complete

${ }^{11} \mathrm{CFT}$ PET/MR scanning because of the impact of COVID-19. One HC had contraindications for PET/MR scanning. Then, 33 participants (17 patients with $\mathrm{MOH}$ and $16 \mathrm{HCs}$ ) completed ${ }^{11} \mathrm{CFT}$ PET/MR. The majority of patients $(16 / 17,94.1 \%)$ overused combination analgesics $(94.1 \%)$, and only one overused triptan (1/17, $5.9 \%$ ). Finally, we included 16 patients who overused combination analgesics for further analysis (Fig. 1). At the two-year follow-up, $11 \mathrm{MOH}$ patients (11/16) reported satisfactory improvement, and 3 of them $(3 / 11)$ relapsed within 1 year.

\section{Participant characteristics}

Participant information is displayed in Table 1. The average age of the $\mathrm{MOH}$ patients $(13 \mathrm{~F} / 3 \mathrm{M})$ was $45.9 \pm$ 7.3 (mean \pm SD) years, with an average duration of headache of $22.9 \pm 8.4$ years and medication overuse of $3.1 \pm 2.9$ years. The mean headache frequency and headache intensity were $22.9 \pm 6.9$ attacks per month and $7.6 \pm 1.0$ (visual analogue scale 1-10), respectively. The mean age of the HCs (12 F/4 M) was $43.1 \pm$ 
6.7 years. Three patients had a nonmigraine-like headache attack when PET scans were performed. Age and sex showed no significant differences between the two groups $(P>0.05)$. Clinical details are provided in Table 2.

Table 1

Participant characteristics

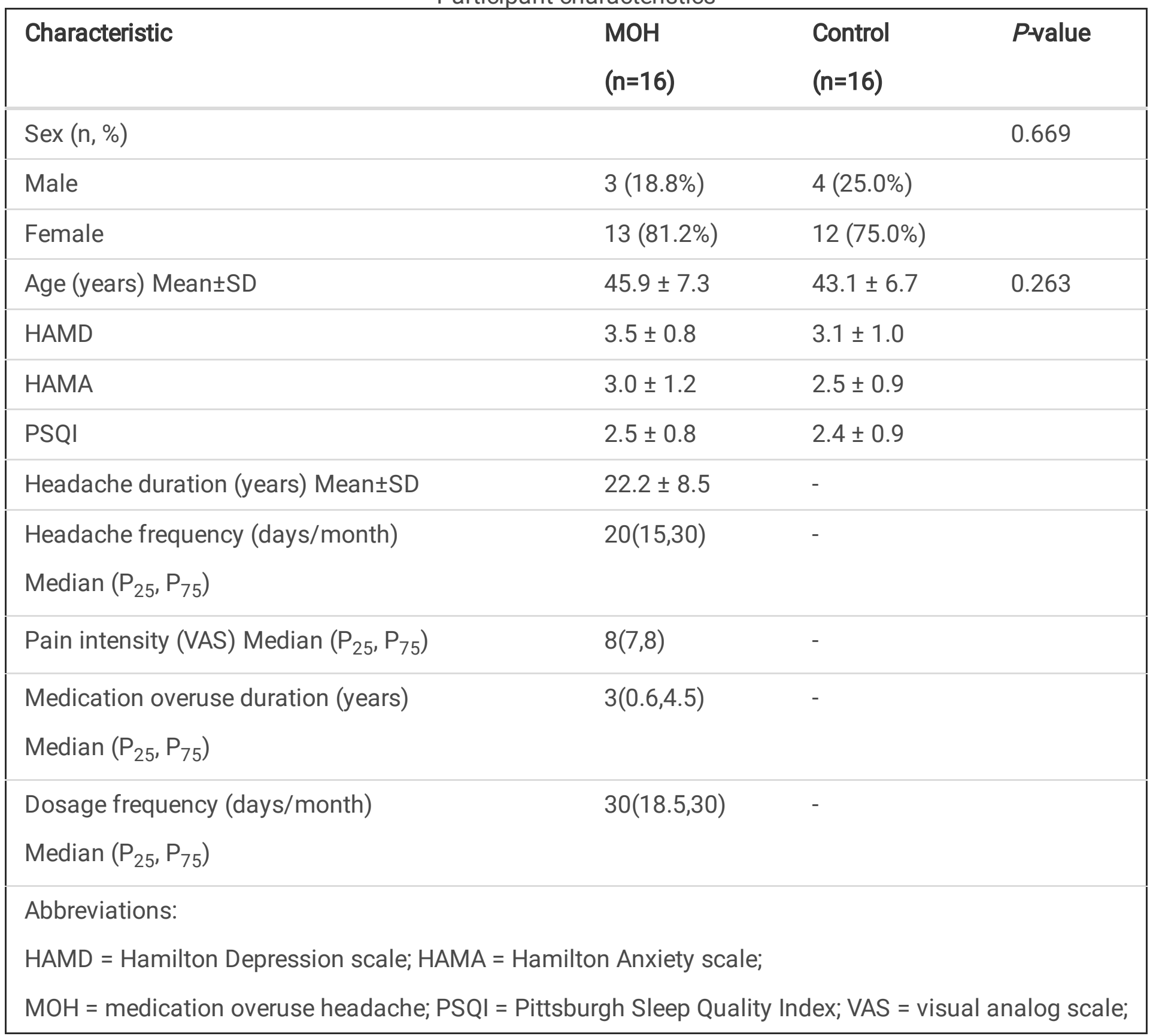


Table 2

Clinical characteristics of $\mathrm{MOH}$ patients $(\mathrm{n}=17)$

\begin{tabular}{|c|c|c|c|c|c|c|c|c|c|}
\hline Patient & $\begin{array}{l}\text { Age } \\
\text { (years) }\end{array}$ & Sex & $\begin{array}{l}\text { Headache } \\
\text { duration } \\
\text { (years) }\end{array}$ & $\begin{array}{l}\text { Medication } \\
\text { overuse } \\
\text { duration } \\
\text { (years) }\end{array}$ & VAS & HAMD & HAMA & PSQI & $\begin{array}{l}\text { Medication } \\
\text { type }\end{array}$ \\
\hline N0.1 & 56 & $\mathrm{~F}$ & 10 & 4 & 7 & 3 & 1 & 3 & $\begin{array}{l}\text { Combination } \\
\text { analgesics }\end{array}$ \\
\hline NO. 2 & 54 & $M$ & 44 & 4 & 8 & 4 & 2 & 2 & $\begin{array}{l}\text { Combination } \\
\text { analgesics }\end{array}$ \\
\hline NO.3 & 45 & $\mathrm{~F}$ & 15 & 8 & 7 & 2 & 3 & 3 & $\begin{array}{l}\text { Combination } \\
\text { analgesics }\end{array}$ \\
\hline NO.4 & 45 & $\mathrm{~F}$ & 15 & 0.5 & 8 & 3 & 3 & 2 & $\begin{array}{l}\text { Combination } \\
\text { analgesics }\end{array}$ \\
\hline N0.5 & 46 & $\mathrm{~F}$ & 26 & 10 & 7 & 4 & 2 & 3 & $\begin{array}{l}\text { Combination } \\
\text { analgesics }\end{array}$ \\
\hline N0.6 & 47 & $F$ & 16 & 0.25 & 7 & 2 & 4 & 1 & $\begin{array}{l}\text { Combination } \\
\text { analgesics }\end{array}$ \\
\hline N0.7 & 38 & $M$ & 25 & 0.6 & 10 & 4 & 5 & 2 & $\begin{array}{l}\text { Combination } \\
\text { analgesics }\end{array}$ \\
\hline NO.8 & 35 & $M$ & 18 & 0.5 & 7 & 4 & 1 & 3 & $\begin{array}{l}\text { Combination } \\
\text { analgesics }\end{array}$ \\
\hline NO.9 & 30 & $\mathrm{~F}$ & 20 & 5 & 6 & 3 & 4 & 4 & $\begin{array}{l}\text { Combination } \\
\text { analgesics }\end{array}$ \\
\hline N0.10 & 48 & $M$ & 12 & 3 & 8 & 3 & 3 & 2 & Triptan \\
\hline N0.11 & 53 & $\mathrm{~F}$ & 30 & 3 & 8 & 4 & 3 & 1 & $\begin{array}{l}\text { Combination } \\
\text { analgesics }\end{array}$ \\
\hline N0.12 & 50 & $\mathrm{~F}$ & 30 & 3 & 8 & 4 & 2 & 3 & $\begin{array}{l}\text { Combination } \\
\text { analgesics }\end{array}$ \\
\hline N0.13 & 48 & $\mathrm{~F}$ & 15 & 5 & 7 & 3 & 3 & 3 & $\begin{array}{l}\text { Combination } \\
\text { analgesics }\end{array}$ \\
\hline N0.14 & 47 & $\mathrm{~F}$ & 30 & 3 & 8 & 5 & 4 & 3 & $\begin{array}{l}\text { Combination } \\
\text { analgesics }\end{array}$ \\
\hline N0.15 & 48 & $\mathrm{~F}$ & 23 & 0.5 & 7 & 3 & 4 & 2 & $\begin{array}{l}\text { Combination } \\
\text { analgesics }\end{array}$ \\
\hline N0.16 & 54 & $\mathrm{~F}$ & 25 & 1 & 8 & 4 & 5 & 2 & $\begin{array}{l}\text { Combination } \\
\text { analgesics }\end{array}$ \\
\hline N0.17 & 39 & $\mathrm{~F}$ & 24 & 0.7 & 9 & 4 & 2 & 3 & $\begin{array}{l}\text { Combination } \\
\text { analgesics }\end{array}$ \\
\hline
\end{tabular}




\begin{tabular}{|c|c|c|c|c|c|c|c|c|c|}
\hline Patient & $\begin{array}{l}\text { Age } \\
\text { (years) }\end{array}$ & Sex & $\begin{array}{l}\text { Headache } \\
\text { duration } \\
\text { (years) }\end{array}$ & $\begin{array}{l}\text { Medication } \\
\text { overuse } \\
\text { duration } \\
\text { (years) }\end{array}$ & VAS & HAMD & HAMA & PSQI & $\begin{array}{l}\text { Medication } \\
\text { type }\end{array}$ \\
\hline \multicolumn{10}{|c|}{ Abbreviations: } \\
\hline \multicolumn{10}{|c|}{ HAMD = Hamilton Depression scale; HAMA = Hamilton Anxiety scale; } \\
\hline \multicolumn{10}{|c|}{$\mathrm{MOH}=$ medication overuse headache; PSQI = Pittsburgh Sleep Quality Index; VAS = visual analog scale } \\
\hline
\end{tabular}

\section{Imaging data}

The SUVr in the left orbitofrontal cortex (OFC; Brodmann 11) was significantly lower in the $16 \mathrm{MOH}$ patients who overused combination analgesics than in the HCs ( $\left.P^{G R F}<0.01\right)$ (Fig. 2). The partial correlation analysis demonstrated no significant correlations between the SUVr in the left OFC and the clinical measures in the patients with $\mathrm{MOH}$.

We subsequently set the left OFC as the seed region to compute RSFC to identify the altered resting-state functional architecture in the $\mathrm{MOH}$ patients compared with the HCs. There was decreased RSFC between the left OFC and the left superior temporal pole (Brodmann 28) and increased RSFC between the left OFC and the bilateral calcarine gyri (Brodmann 17; primary visual cortex) (Fig. 3). There were no significant correlations between the connectivity strength linking the positive brain regions and the left OFC and clinical measures in the $\mathrm{MOH}$ patients.

\section{Discussion}

To the best of our knowledge, this is the first PET/MR study to examine abnormalities in DAT availability in the brains of $\mathrm{MOH}$ subjects, and decreased DAT availability in the left OFC was observed. We also found that there was altered FC between the left OFC and the left superior temporal pole and the bilateral calcarine gyri.

We excluded possible potential confounding factors, including depression, anxiety, and sleep disturbance, in our study for the following reasons. First, a previous study found that DAT availability in the striatum was lower in individuals with psychiatric comorbidities such as major depressive disorder [9]. Moreover, recent findings have indicated that the mesocorticolimbic pathway comprising the ventral tegmental area and the nucleus accumbens is involved in controlling sleep and wakefulness [16]. Rodent studies have also revealed that ventral tegmental area dopamine neurons play a wake-regulatory role [16]. We believe it is worth evaluating the impact of medication overuse and chronic headache on DAT availability in the mesocorticolimbic dopamine system. However, the strict recruitment criteria resulted in the relatively small sample size in our study because $\mathrm{MOH}$-associated psychiatric comorbidities are very common. ${ }^{17}$ In addition, previous studies in China showed that combination analgesics were the most commonly overused medications $[14,18]$. In contrast, overuse of triptans is very rare in China, as was found earlier in other regions $[19,20,21]$. Therefore, patients overusing combination analgesics were enrolled in the final analysis in the present study. 
We first found that $\mathrm{MOH}$ patients exhibited decreased DAT availability in the OFC, which is involved in both drug addiction and pain processing. The OFC has been suggested to play a key role in drug addiction by behavioural and pharmacological studies [7]. A previous FDG-PET study also found persistent hypometabolism in the OFC in $\mathrm{MOH}$ patients before and 3 weeks after detoxification and therefore confirmed that $\mathrm{MOH}$ was related to drug addiction [22]. In addition, dopamine is a vital neurotransmitter involved in drug addiction, and previous studies have verified that patients with drug addiction present abnormalities in the dopamine pathway [8]. Taken together, the reduction in DAT availability in the OFC observed in our study could be interpreted as direct evidence that drug addiction is involved in the pathogenesis of $\mathrm{MOH}$. On the other hand, the decreased DAT availability of the OFC could also be related to pain processing (Fig. 4). Glutamatergic projections from the ventrolateral OFC to the periaqueductal grey (PAG) are involved in antinociception in rats [23]. The projection neurons could be inhibited by GABAergic neurons, which in turn are inhibited by opioids or dopamine [24]. Lower DAT availability in the OFC in $\mathrm{MOH}$ patients implied lower dopamine transmitter levels in the OFC. Reductions in dopamine would reduce the inhibition of GABAergic neurons, which increases the activation of GABAergic neurons and decreases activity in the PAG. The PAG is the region with primary control over descending pain modulation and pain relief [25]. Thus, lower dopamine levels in the OFC decreasing the activity in the PAG could result in decreased inhibitory pain modulation and facilitate the process of central sensitization, leading to chronic headaches [23]. Therefore, we speculate that the OFC is involved in the pathogenesis of $\mathrm{MOH}$, which may be related to its roles in both addiction and pain.

We observed decreased DAT availability located in the left rather than right OFC in MOH patients, which may reveal functional differences. Previous studies with $\mathrm{MOH}$ patients demonstrated that cortical thickness was lower in the left prefrontal cortex [26], and the grey matter in the left OFC was nonsignificantly decreased [27], implying that there may be a difference between the left and right OFC in the pathogenesis of $\mathrm{MOH}$. Lopez-Persem et al. first identified an asymmetrical response of the lateral OFC to reward in human and nonhuman primates [28]. A Neurosynth meta-analysis suggested that value-guided and motivational processes might be supported more by the left OFC, while the right OFC has a greater role in emotional and affective processes [28]. In addition, a recent voxel-based morphometry study revealed that individuals with more grey matter volume (GMV) in the left OFC tended to be less impulsive and more goal-directed [29], which helps individuals make better decisions. Therefore, it is understandable that dysfunction of the left OFC may make patients more prone to overuse analgesics. Taken together, we speculate that the left OFC might play a more important role than the right OFC in the pathogenesis of $\mathrm{MOH}$, which provides a precise target for the treatment of $\mathrm{MOH}$ in the future.

The partial correlation analysis demonstrated no significant correlations between the left OFC SUVr and the clinical measures in patients with $\mathrm{MOH}$ after correcting for age and sex. Because only $3 \mathrm{MOH}$ patients relapsed during the two-year follow-up, we did not perform an analysis between $\mathrm{MOH}$ relapse and left OFC SUVr. Although previous studies have demonstrated that cortical volume [30] and metabolism [22] in the OFC were related to treatment response and relapse, these studies used different imaging methods to investigate the correlation between aspects of the OFC and clinical measures; thus, the results may not be directly comparable. Further studies are needed to explore the potential factors that affect the reduction in DAT in the OFC. 
Further functional analysis revealed that there was altered FC between the left OFC and the left superior temporal pole and the bilateral calcarine gyri. Previous structural MRI studies demonstrated that the left temporal pole showed increased GMV in $\mathrm{MOH}$ patients with chronic migraine [31]. One diagnosis classification study of migraine proposed that the structural characteristics (such as cortical thickness and volume) of the temporal pole could serve as one of the principal classifiers [32], indicating that the temporal cortex might play a crucial role in the pathogenesis of migraine and $\mathrm{MOH}$. Furthermore, functional MRI coupled with noxious thermal stimulation revealed that the temporal polar region was also associated with an increased frequency of migraine attacks [33]. Therefore, decreased FC between the left OFC and left temporal polar cortex confirmed in our study may suggest associated dysfunction in pain modulation present in $\mathrm{MOH}$ patients. Cortical spreading depression may originate from the visual cortex $[34,35,36]$ and can activate trigeminal nociception and thus trigger headache [36]. Previous studies have found higher local gyrification in the right occipital pole [26] and decreased GMV in occipital areas in $\mathrm{MOH}$ patients compared with controls [31], indicating the involvement of the visual cortex in $\mathrm{MOH}$. In a rat model of $\mathrm{MOH}$, exposure to sumatriptan for two weeks produced long-lasting increased susceptibility to evoked cortical spreading depression [37]. In addition, the visual cortex has been demonstrated to be involved in the mesocorticolimbic dopamine system [38, 39]. Decreased GMV and altered functional activity in the visual cortex have been reported in patients with dependence problems such as gambling disorder and cocaine addiction [40, 41]. Therefore, we speculate that increased FC between the left OFC and bilateral visual cortex was correlated with the development of $\mathrm{MOH}$ through its role in drug addiction and headache. Unfortunately, we did not find any significant correlation between the connectivity strength linking the positive brain regions with the left $\mathrm{OFC}$ and the clinical measures in the $\mathrm{MOH}$ patients.

A number of potential limitations need to be considered. First, the sample size of the $\mathrm{MOH}$ patients was relatively small because of strict enrolment criteria. Second, as $94.1 \%(16 / 17)$ of the $\mathrm{MOH}$ patients overused combination analgesics in our present study, it was difficult to perform a subgroup analysis to differentiate the effects of different analgesics on DAT availability in the OFC. Moreover, we did not enrol patients with chronic migraine without medication overuse, because it was difficult to recruit "pure" chronic migraine patients without anxiety, depression, sleep disorders or medication overuse $[42,43]$. Therefore, it is difficult to directly answer the question of whether the decreased DAT availability in the left OFC was secondary to protracted drug self-administration, chronic headache or both. Finally, due to the influence of COVID-19 and the patient's willingness to participate in the re-examination, most of the $\mathrm{MOH}$ patients who successfully abstained from the drug did not complete the re-examination with ${ }^{11} \mathrm{CFT} P E T / M R$, resulting in unclear changes in the DAT in the OFC after withdrawal.

\section{Conclusions}

The reduction in DAT availability in the left OFC in $\mathrm{MOH}$ patients who overused combination analgesics provides in vivo molecular imaging evidence that the left OFC, as a part of the mesocorticolimbic dopamine system, directly participates in the pathogenesis of $\mathrm{MOH}$, and this study may cast light on an underlying pharmacological and behavioural treatment target for those with $\mathrm{MOH}$. In addition, the OFC on both sides may have different functions in the pathogenesis of $\mathrm{MOH}$. Altered intrinsic FC with the left OFC was identified in $\mathrm{MOH}$ patients, which provides a new perspective to understand the pathogenesis of $\mathrm{MOH}$. 


\section{Abbreviations}

${ }^{11} \mathrm{CFT}:{ }^{11} \mathrm{C}-2 \beta$-carbomethoxy-3 $\beta$-(4-fluorophenyl) tropan; DAT: dopamine transporter; FC: functional connectivity; FWHM: full-width at half-maximum; GRF: Gaussian random field; GMV: grey matter volume; HCs: healthy controls; ICHD-3: International Classification of Headache Disorders $-3^{\text {rd }}$ edition; $\mathrm{MOH}$ : medication overuse headache; OFC: orbitofrontal cortex; PAG: periaqueductal grey; RSFC: resting-state functional connectivity; SUVr: standardized uptake value ratio.

\section{Declarations}

\section{Ethics approval and consent to participate}

This study was approved by the ethics committee of Chinese PLA General Hospital (S2018-027-02) and registered in the Chinese Clinical Trial Registry (ChicTR1800016773). All subjects provided written informed consent in accordance with the Declaration of Helsinki.

\section{Consent for publication}

Not applicable.

\section{Availability of data and materials}

All data generated and analyzed during the current study will be available from the corresponding authors on reasonable request.

\section{Competing interests}

The authors declared that they have no competing interests.

\section{Funding}

This work was supported by grants from the National Natural Science Foundation of China (grant 81771200, 82071226), and Beijing Natural Science Foundation Essential Research Project (Z170002).

\section{Author contributions}

Zhao Dong, Shengyuan Yu, and Huanxian Liu contributed to conception and design of the study, Jiajin Liu, Baixuan Xu and Shuping Sun contributed to acquisition of data,Binbin Nie contribute to analysis of data, Wei Dai contributed to drafting a significant portion of the figures.

\section{Acknowledgements}

We thank all patients and healthy volunteers who took part in the study.

\section{References}


1. Headache Classification Committee of the International Headache Society (2018) The International Classification of Headache Disorders, 3rd edition. Cephalalgia 38(1):1-211.

2. Diener HC, Dodick D, Evers S et al (2019) Pathophysiology, prevention, and treatment of medication overuse headache. The Lancet Neurology 18(9):891-902.

3. GBD 2015 Disease and Injury Incidence and Prevalence Collaborators (2016) Global, regional, and national incidence, prevalence, and years lived with disability for 310 diseases and injuries, 1990-2015: a systematic analysis for the Global Burden of Disease Study 2015. Lancet 388(10053):1545-1602.

4. Saper JR, Hamel RL, Lake AE 3rd (2005) Medication overuse headache ( $\mathrm{MOH})$ is a biobehavioural disorder. Cephalalgia 25(7):545-546.

5. Calabresi P, Cupini LM (2005) Medication-overuse headache: similarities with drug addiction. Trends in pharmacological sciences 26(2):62-68.

6. Solinas M, Belujon P, Fernagut PO et al (2019) Dopamine and addiction: what have we learned from 40 years of research. J Neural Transm (Vienna) 126(4):481-516.

7. Volkow ND, Fowler JS, Wang GJ (2004) The addicted human brain viewed in the light of imaging studies: brain circuits and treatment strategies. Neuropharmacology 47 Suppl 1:3-13.

8. Volkow ND (2004) Imaging the addicted brain: from molecules to behavior. Journal of nuclear medicine $45(11): 13 \mathrm{~N}-16 \mathrm{~N}, 19 \mathrm{~N}-20 \mathrm{~N}, 22 \mathrm{~N}$ passim.

9. Pizzagalli DA, Berretta S, Wooten D et al (2019) Assessment of Striatal Dopamine Transporter Binding in Individuals With Major Depressive Disorder: In Vivo Positron Emission Tomography and Postmortem Evidence. JAMA Psychiatry 76(8):854-861.

10. Lucas LR, Celen Z, Tamashiro KL et al (2004) Repeated exposure to social stress has long-term effects on indirect markers of dopaminergic activity in brain regions associated with motivated behavior. Neuroscience 124(2):449-457.

11. Brake WG, Zhang TY, Diorio J et al (2004) Influence of early postnatal rearing conditions on mesocorticolimbic dopamine and behavioural responses to psychostimulants and stressors in adult rats. The European journal of neuroscience 19(7):1863-1874.

12. Lucas LR, Wang CJ, McCall TJ et al (2007) Effects of immobilization stress on neurochemical markers in the motivational system of the male rat. Brain research 1155:108-115.

13. Yamamoto $Y$, Takahata K, Kubota M et al (2021) Differential associations of dopamine synthesis capacity with the dopamine transporter and D2 receptor availability as assessed by PET in the living human brain. Neuroimage 226:117543. 
14. Dong Z, Chen X, Steiner TJ et al (2015) Medication-overuse headache in China: clinical profile, and an evaluation of the ICHD-3 beta diagnostic criteria. Cephalalgia 35(8):644-651.

15. Yan CG, Wang XD, Zuo XN et al (2016) DPABI: Data Processing \& Analysis for (Resting-State) Brain Imaging. Neuroinformatics 14(3):339-351.

16. Oishi Y, Lazarus M (2017) The control of sleep and wakefulness by mesolimbic dopamine systems. Neurosci Res 118:66-73.

17. Atasoy HT, Atasoy N, Unal AE et al (2005) Psychiatric comorbidity in medication overuse headache patients with pre-existing headache type of episodic tension-type headache. European journal of pain 9(3):285-291.

18. Wang Y, Zhou J, Fan X et al (2011) Classification and clinical features of headache patients: an outpatient clinic study from China. The journal of headache and pain 12(5):561-567.

19. Lu SR, Fuh JL, Chen WT et al (2001) Chronic daily headache in Taipei, Taiwan: prevalence, follow-up and outcome predictors. Cephalalgia 21(10):980-986.

20. Kanki R, Nagaseki Y, Sakai F (2008) Medication-overuse headache in Japan. Cephalalgia 28(11):12271228.

21. Imai N, Kitamura E, Konishi T et al (2007) Clinical features of probable medication-overuse headache: a retrospective study in Japan. Cephalalgia 27(9):1020-1023.

22. Fumal A, Laureys S, Di Clemente $L$ et al (2006) Orbitofrontal cortex involvement in chronic analgesicoveruse headache evolving from episodic migraine. Brain 129(Pt 2):543-550.

23. Huang J, Zhang Z, Gambeta E et al (2021) An orbitofrontal cortex to midbrain projection modulates hypersensitivity after peripheral nerve injury. Cell Rep 35(4):109033.

24. Ong WY, Stohler CS, Herr DR (2019) Role of the Prefrontal Cortex in Pain Processing. Molecular neurobiology 56(2):1137-1166.

25. Goadsby PJ, Holland PR, Martins-Oliveira M et al (2017) Pathophysiology of Migraine: A Disorder of Sensory Processing. Physiological reviews 97(2):553-622.

26. Riederer F, Schaer M, Gantenbein AR et al (2017) Cortical Alterations in Medication-Overuse Headache. Headache 57(2):255-265.

27. Schmidt-Wilcke T, Leinisch E, Straube A et al (2005) Gray matter decrease in patients with chronic tension type headache. Neurology 65(9):1483-1486.

28. Lopez-Persem A, Roumazeilles L, Folloni D et al (2020) Differential functional connectivity underlying asymmetric reward-related activity in human and nonhuman primates. Proceedings of the National Academy of Sciences of the United States of America 117(45):28452-28462. 
29. Zhang R, Chen Z, Xu T, Feng T (2021) The neural basis underlying the relation between the action identification level and delay discounting: The medial and orbital frontal cortex functional connectivity with the precuneus. Int J Psychophysiol 159:74-82.

30. Riederer F, Gantenbein AR, Marti M et al (2013) Decrease of gray matter volume in the midbrain is associated with treatment response in medication-overuse headache: possible influence of orbitofrontal cortex. The Journal of neuroscience 33(39):15343-15349.

31. Lai TH, Chou KH, Fuh JL et al (2016) Gray matter changes related to medication overuse in patients with chronic migraine. Cephalalgia 36(14):1324-1333.

32. Schwedt TJ, Chong CD, Wu T et al (2015) Accurate Classification of Chronic Migraine via Brain Magnetic Resonance Imaging. Headache 55(6):762-777.

33. Maleki N, Becerra L, Brawn J et al (2012) Concurrent functional and structural cortical alterations in migraine. Cephalalgia 32(8):607-620.

34. Schwedt TJ, Dodick DW (2009) Advanced neuroimaging of migraine. The Lancet Neurology 8(6):560568.

35. Charles A, Brennan K (2009) Cortical spreading depression-new insights and persistent questions. Cephalalgia 29(10):1115-1124.

36. Pietrobon D, Moskowitz MA (2013) Pathophysiology of migraine. Annual review of physiology 75:365391.

37. Green AL, Gu P, De Felice M et al (2014) Increased susceptibility to cortical spreading depression in an animal model of medication-overuse headache. Cephalalgia 34(8):594-604.

38. Arsenault JT, Nelissen K, Jarraya B et al (2013) Dopaminergic reward signals selectively decrease fMRI activity in primate visual cortex. Neuron 77(6):1174-1186.

39. Hanlon CA, Dowdle LT, Naselaris T (2014) Visual cortex activation to drug cues: a meta-analysis of functional neuroimaging papers in addiction and substance abuse literature. Drug Alcohol Depend 143:206212.

40. Meng YJ, Deng W, Wang HY et al (2014) Reward pathway dysfunction in gambling disorder: A metaanalysis of functional magnetic resonance imaging studies. Behav Brain Res 275:243-251.

41. van Wingen GA, van den Brink W, Veltman DJ et al (2013) Reduced striatal brain volumes in nonmedicated adult ADHD patients with comorbid cocaine dependence. Drug Alcohol Depend 131(3):198-203.

42. Wakerley BR (2019) Medication-overuse headache. Pract Neurol 19(5):399-403.

43. Evers S, Marziniak M (2010) Clinical features, pathophysiology, and treatment of medication-overuse headache. The Lancet Neurology 9(4):391-401. 


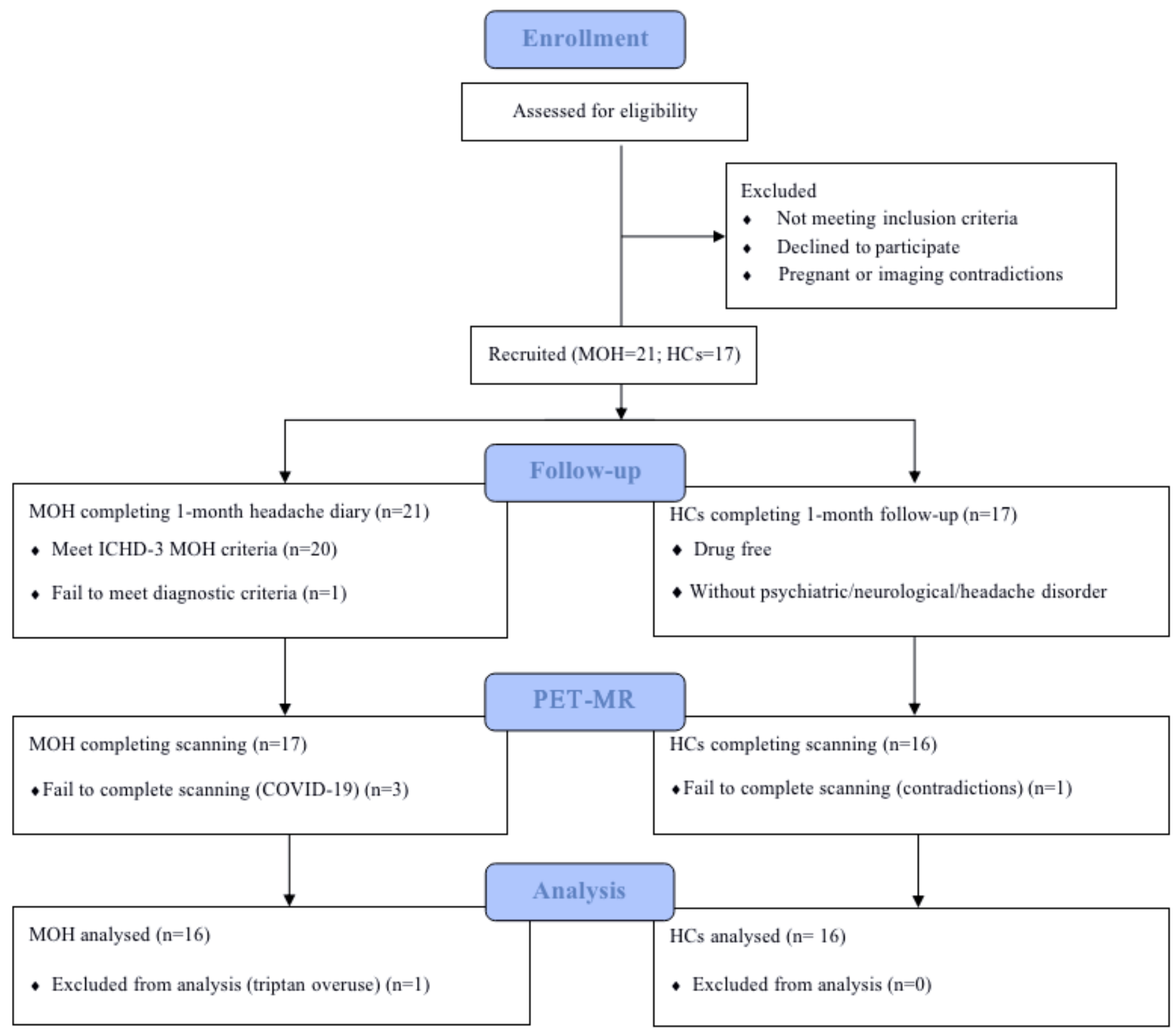

\section{Figure 1}

Study design. $\mathrm{MOH}=$ medication overuse headache; $\mathrm{HCs}=$ healthy controls. 


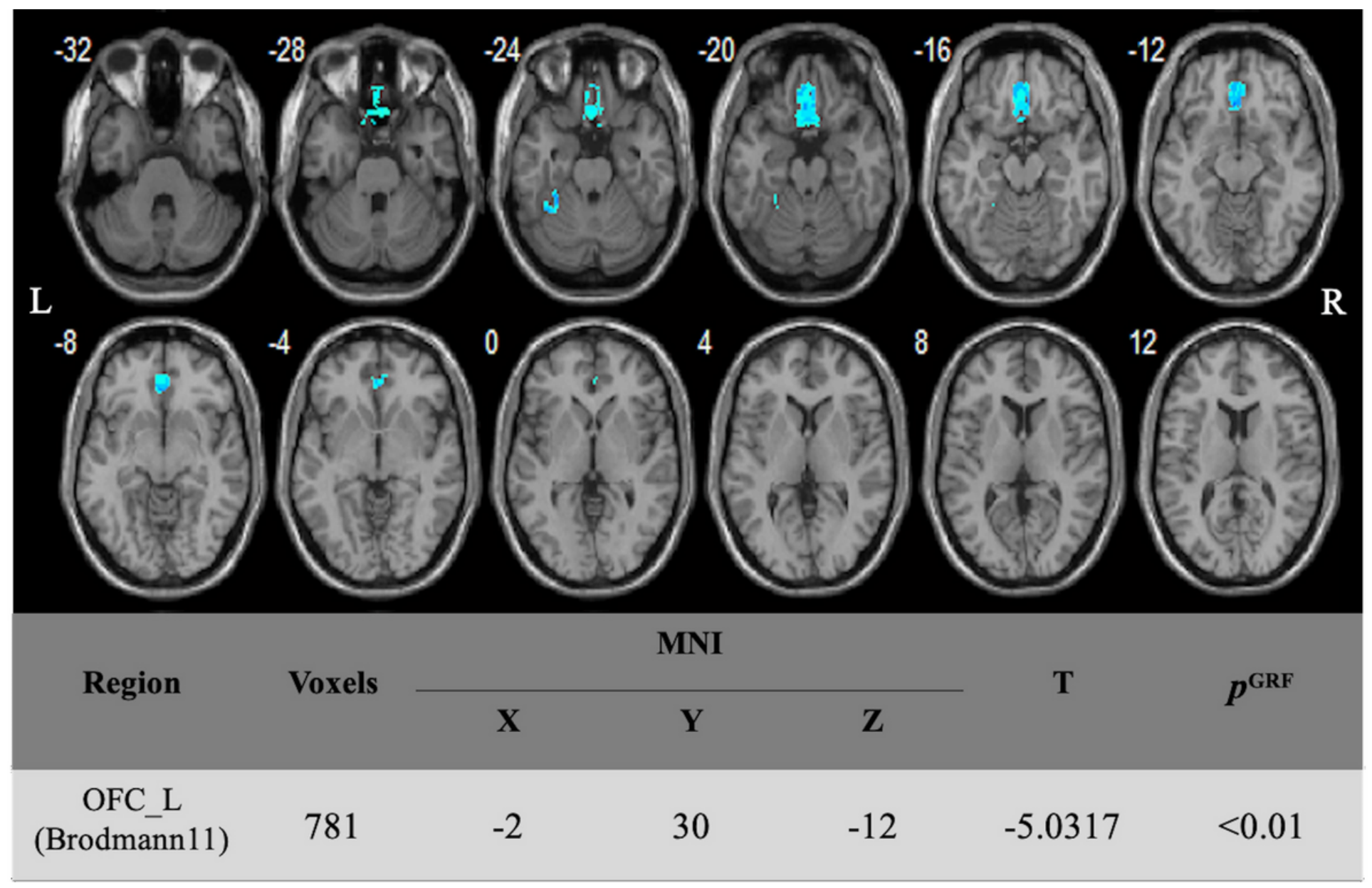

\section{Figure 2}

Area in which the standardized uptake value ratio (SUVr) was lower in $\mathrm{MOH}$ patients than in controls. The region of decreased 11CFT SUVr in the $\mathrm{MOH}$ patients compared to the controls is shown in blue. Group differences in cortical 11CFT SUVr projected on transverse sections of a normalized brain MRI template. MNI = Montreal Neurological Institute, X, Y, Z coordinates of the primary; GRF = Gaussian random field; $\mathrm{MOH}$ : medication overuse headache; OFC_L = left orbitofrontal cortex. 


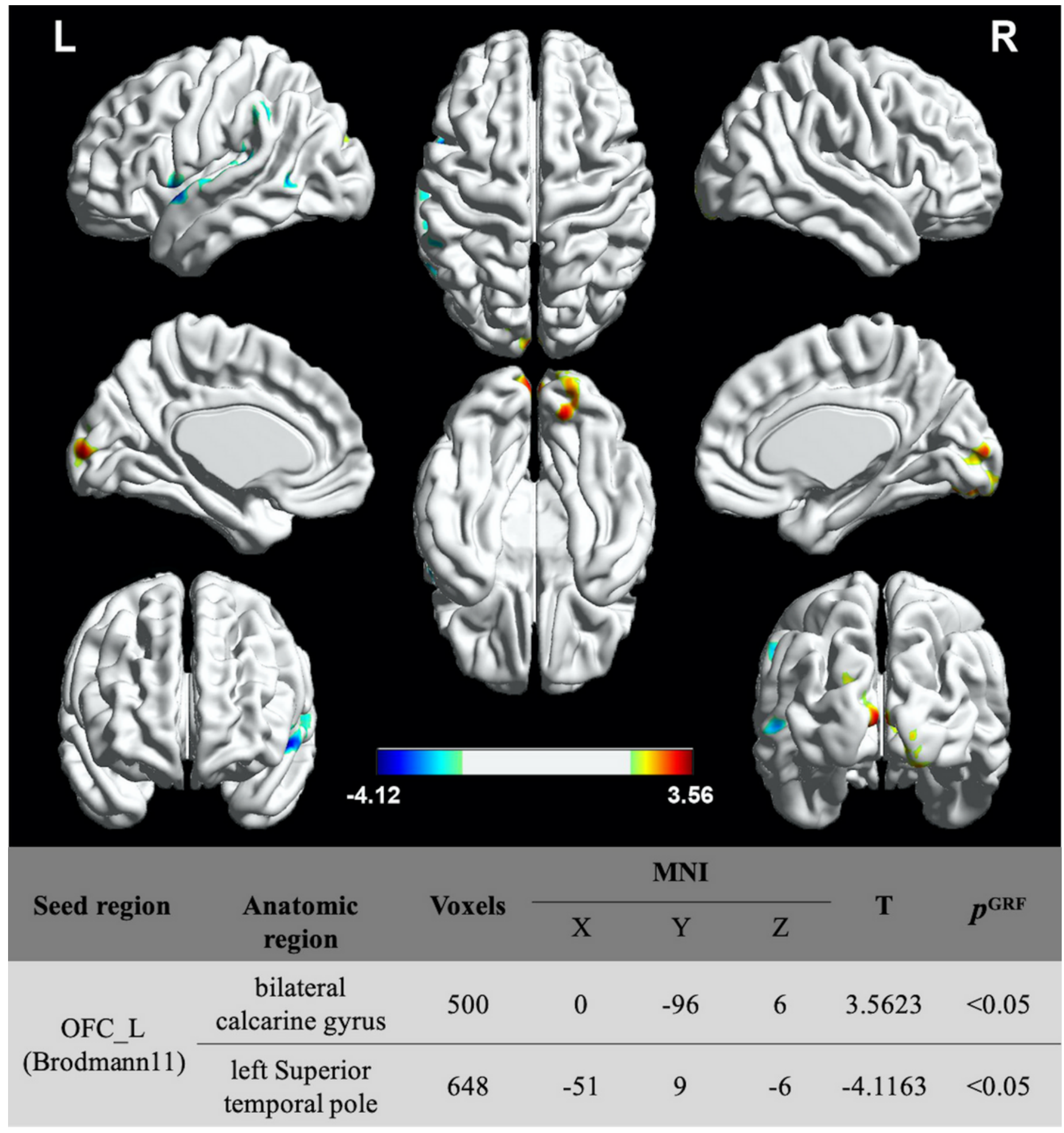

\section{Figure 3}

Altered RSFC in the left OFC. Decreased RSFC between the left OFC and the left superior temporal pole (blue), and increased RSFC between the left OFC and the bilateral calcarine gyri (red) in the $\mathrm{MOH}$ patients compared to the controls. RSFC = resting-state functional connectivity; OFC_L = left orbitofrontal cortex; $\mathrm{MNI}$ $=$ Montreal Neurological Institute, $\mathrm{X}, \mathrm{Y}, \mathrm{Z}$ coordinates of the primary; GRF = Gaussian random field; $\mathrm{MOH}=$ medication overuse headache. 


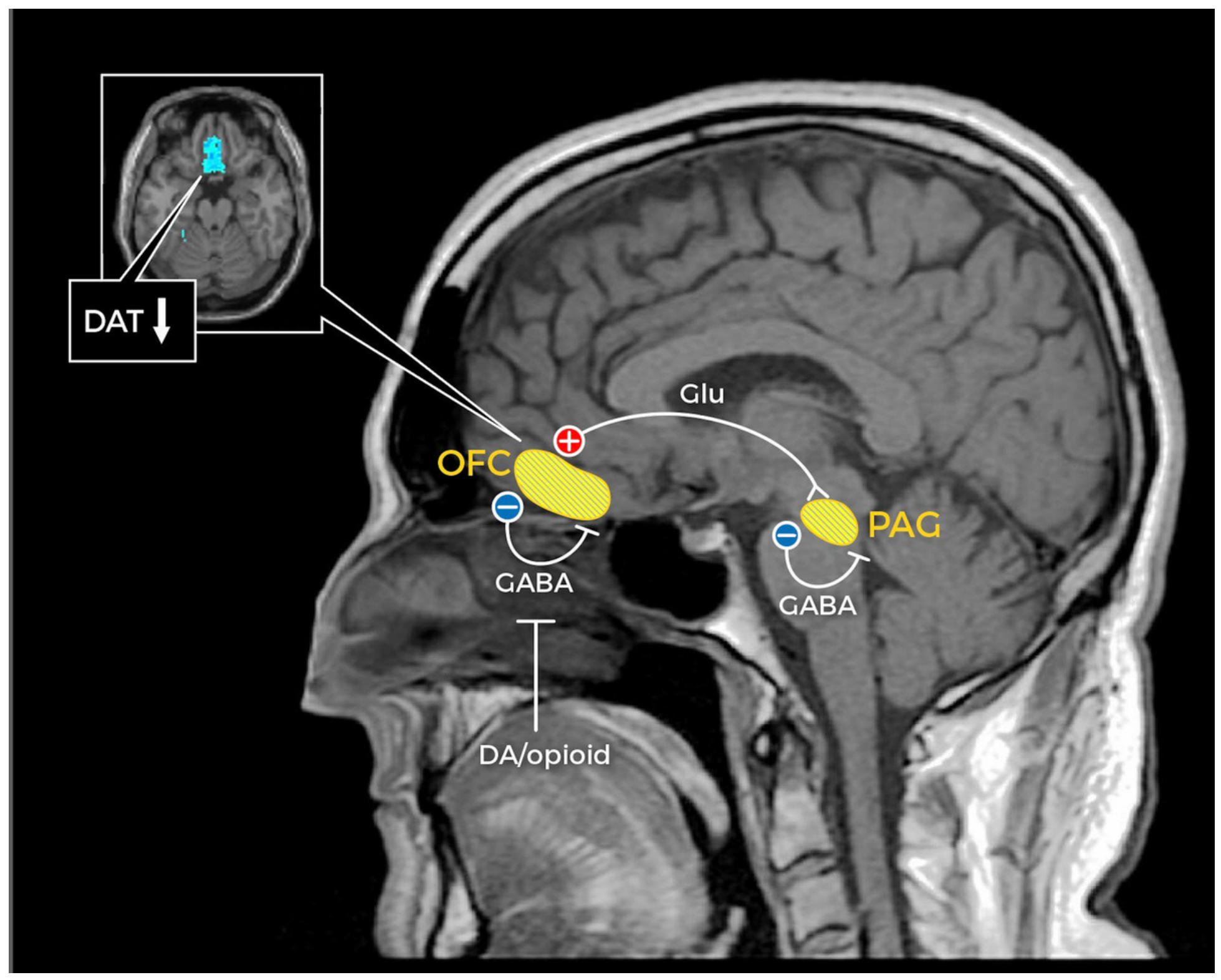

\section{Figure 4}

Schematic diagram of chronic headache caused by low availability of dopamine transporters. Glutamatergic projections from the ventrolateral OFC to the periaqueductal grey are inhibited by GABAergic neurons, which in turn is inhibited by opioids or dopamine. DA = dopamine; DAT = dopamine transporter; GABA = Gammaaminobutyric acid; Glu = glutamic acid; OFC = orbitofrontal cortex; PAG = periaqueductal grey.

\section{Supplementary Files}

This is a list of supplementary files associated with this preprint. Click to download.

- STROBEchecklistcrosssectional.docx 